\title{
Efficacy and safety of duloxetine in osteoarthritis: a systematic review and meta-analysis
}

\author{
Mikala C. Osani and Raveendhara R. Bannuru
}

Center for Treatment Comparison and Integrative Analysis (CTCIA), Division of Rheumatology, Tufts Medical Center, Boston, MA, USA

Received: December 21, 2018 Accepted: January 7, 2019

\section{Correspondence to}

Raveendhara R. Bannuru, M.D. Center for Treatment Comparison and Integrative Analysis (CTCIA), Division of Rheumatology, Tufts Medical Center, Box 406, 800 Washington St, Boston, MA 02111, USA

Tel: $+1-617-636-8284$

Fax: +1-617-636-1542

E-mail:rbannuru@

tuftsmedicalcenter.org
About $21 \%$ of adults with osteoarthritis (OA) are diagnosed with concomitant depression in addition to chronic pain. Duloxetine, an anti-depressant medication, has been recently approved for managing Knee OA. We performed a systematic review to ascertain the efficacy and safety of duloxetine for OA. We searched MEDLINE, EMBASE, Web of Science, Google Scholar, and the Cochrane Database from inception to December 2018. Randomized clinical trials (RCTs) assessing the efficacy and/or safety of duloxetine versus placebo in OA patients were included. Data extraction and quality assessment were undertaken by two independent reviewers. Seven RCTs ( $\mathrm{n}=2,102$ participants) met our inclusion criteria, and five RCTs $(n=1,713)$ were eligible for meta-analysis. The results of our analyses indicate that duloxetine has statistically significant, moderate benefits on pain, function, and quality of life in knee OA patients for up to 13 weeks. Reported incidences of gastrointestinal adverse events were three to four times higher in participants who received duloxetine versus placebo. Duloxetine may be an effective treatment option for individuals with knee OA, but use of the drug is associated with a significantly higher risk of adverse events. Patient preferences and clinicians' judgment must be considered before the initiation of duloxetine.

Keywords: Osteoarthritis; Duloxetine; Chronic pain; Depression; Meta-analysis

\section{INTRODUCTION}

Osteoarthritis (OA) is one of the most common causes of chronic pain in adults worldwide [1-3]. Elderly Korean adults with OA report significantly higher pain scores than their peers, and experience significant declines in functional capacity and quality of life as a result of the disease [4]. The chronic pain experienced by individuals with OA has been partially attributed to a biological process called central sensitization, which may be driven by painful stimuli that originate from damaged bone and joint tissue [5]. Central sensitization has been observed both in animal and human models of OA [6-8]. In addition to chronic pain and disability, approximately $21 \%$ of adults with OA are diagnosed with concomitant depression [9]. Individuals who experience OA with comorbid depression report higher healthcare utilization costs than those without, and they have been shown to use pain medication more frequently [10]. These individuals are also less likely to practice adequate adherence to recommended treatment regimens than patients with OA who have not been diagnosed with depression [10]. Duloxetine is a serotonin and norepinephrine reuptake inhibitor that has demonstrated effective relief of persistent pain in animal models of central sensitization in OA, and randomized clinical trial (RCT) data in OA patients have shown promising results for symptom relief [8]. We conducted a systematic review and meta-analysis to assess the safety and efficacy of Duloxetine in patients with $\mathrm{OA}$. 


\section{DATA SOURCES/SEARCHES}

We conducted a systematic search of the MEDLINE, EMBASE, Google Scholar, Web of Science, and the Cochrane Central Register of Controlled Trials from inception to December 2018 (Appendix 1). Reference lists of relevant systematic reviews and supplements of conference proceedings published up until December 2018 were reviewed by hand. We limited the search to RCTs in human subjects with OA of any joint location, but did not place any limits on language, publication date, or publication status (Supplementary Table 1) [11].

\section{STUDY SELECTION}

RCTs that assessed the efficacy of duloxetine versus a matching placebo in participants with a clinical diagnosis of OA were considered eligible. Among these, we included studies that reported data from validated scales assessing pain, functional status, depression, and quality of life, as well as data on adverse events. Abstract and full-text screening was performed by two independent reviewers, according to the established inclusion criteria.

\section{DATA EXTRACTION AND QUALITY ASSESS- MENT}

Data from the included RCTs were extracted into RevMan software by two independent reviewers [12]. Information was collected on study characteristics and demographic characteristics of the sample, duloxetine dosing regimen, concomitant medication usage, and rescue medication protocols. Quality assessments were performed independently by two reviewers within RevMan using the Cochrane Risk of Bias tool [13].

\section{Outcome definitions}

The primary outcomes of pain and function assessed by validated scales were collected as mean change from baseline to study follow-up. Secondary outcomes of depression and quality of life assessed by validated scales were also collected as mean change from baseline to study follow-up; a standard directionality of these scales was established a priori so that improvement was reflected in decreases in depression scores and increases in quality of life scores. Discontinuations due to adverse events included any participants who discontinued study medication or ceased participation in the study because of an adverse event, regardless of its association to the study medication. Withdrawals due to adverse events were collected only within the RCT follow-up time. Treatment-emergent adverse events (TEAEs) included any adverse event that originated after the initiation of the study medication; these events may or may not be related to the study medication. Serious adverse events (SAEs) were strictly defined as events that were specifically classified by study personnel as "Serious Adverse Events" within the timeframe of the study. Data on the incidence of gastrointestinal (GI) adverse events was collected because GI symptoms are known side effects of duloxetine [14,15].

\section{Statistical analysis}

We calculated standardized mean differences (SMDs) and $95 \%$ confidence intervals (CI) using the DerSimonian and Laird [16] inverse variance method. In the anticipation of clinical and methodological heterogeneity, we conducted meta-analyses using random effects models [16]. Dichotomous outcomes were analyzed using the Mantel-Haenszel method, and we reported the results as risk ratios (RRs) with 95\% CIs [17]. We measured inconsistency by the $I^{2}$ statistic, and between-trial variance was assessed using Tau squared. Meta-analyses were conducted using RevMan software [18,19].

Sensitivity analyses were conducted by excluding "very low quality" RCTs. "Very low quality" refers to those RCTs that received $\geq 2$ high risk of bias ratings; or one specific high risk rating in the "other" category in addition to $\geq 2$ unclear risk ratings; or $\geq 3$ unclear risk of bias ratings in dimensions other than the "other" category using the Cochrane risk of bias tool RCTs assessing the individual efficacy of various doses/dosing regimens of duloxetine indicate no differences between doses or dosing schedules; therefore, we did not plan separate subgroup analyses to account for these factors [20]. 


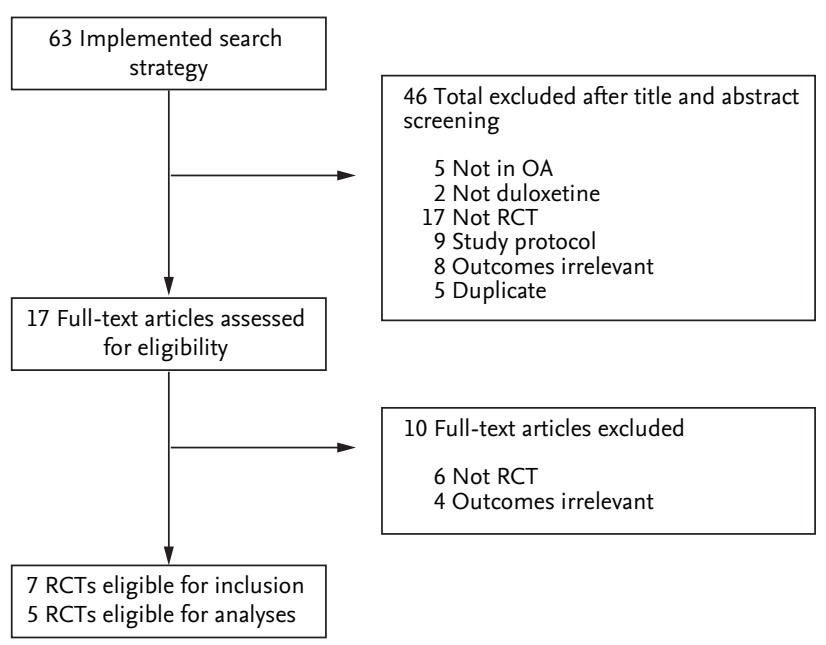

Figure 1. Study flow diagram. OA, osteoarthritis; RCT, randomized clinical trial.

\section{RESULTS}

Our systematic search returned 63 references (Fig. 1). Of these, seven RCTs ( $n=2,102$ participants) comparing duloxetine to a matching placebo met our inclusion criteria [21-27]. Included RCTs were published between 2009 and 2018. The mean age of participants ranged from 60.5 to 68.7 years (median, 62.3), and the proportion of female participants ranged from $57.1 \%$ to $83.7 \%$ (median, 76.7\%). All studies administered duloxetine at $60 \mathrm{mg} /$ day. Seven studies described titration and tapering periods for duloxetine dosing in detail, with three studies allowing for dose modification from 60 to 120 mg/day (Table 1). The majority of studies allowed for limited use of analgesics as rescue medication during the study period. In two studies, continuation of the current regimen of acetaminophen or nonsteroidal anti-inflammatory drug (NSAID) was permitted, and in one study, continuation and optimization of a current NSAID was a part of the study protocol.

Study quality of most RCT's was assessed to be moderate to high; one study received a very low quality rating due to insufficient detail in methodological reporting (Figs. 2 and 3) [24]. High discontinuation rates were observed in over half of the RCTs, and differential discontinuation rates and/or reasons were observed in the duloxetine groups versus placebo groups in three of these cases $[23,24,27]$. Two RCTs were excluded from the final analyses: the research team associated with one RCT had been previously flagged for research misconduct, prompting removal of the study from the analysis; the other RCT was the only study involving participants with hand OA; thus, there was insufficient RCT data to merit meta-analysis $[21,27,28]$.

All five of the studies that were eligible for meta-analysis were conducted in participants with knee OA [2226]. The follow-up time for all studies ranged from 12 to 14 weeks.

\section{Efficacy and safety of duloxetine in knee OA}

\section{Pain and function}

The results of efficacy and safety analyses are displayed in Table 2. Pain was reported in all five RCTs that were eligible for analysis ( $n=1,713$ participants) [22-26]. Duloxetine demonstrated moderate and statistically significant effects on pain reduction over a 12- to 14week follow-up (SMD, -0.38; 95\% CI, -0.48 to -0.28). Sensitivity analysis excluding one very low quality study produced similar results, with an effect size of -0.39 (95\% $\mathrm{CI},-0.52$ to -0.25$)$. Functional improvement was reported in all five RCTs ( $n=1,695$ participants), and duloxetine demonstrated modest, statistically significant benefits (SMD, $-0.35 ; 95 \%$ CI, -0.46 to -0.24 ) within 12 to 14 weeks. The results of sensitivity analysis excluding one very low quality study were no different from the main results (SMD, $-0.33 ; 95 \% \mathrm{CI},-0.47$ to -0.18 ). Assessments of $\mathrm{I}^{2}$ and $\mathrm{T}^{2}$ revealed minimal heterogeneity between studies, for all analyses.

Depression and quality of life

Based on the data from two RCTs ( $\mathrm{n}=584$ ), duloxetine did not show any significant benefits on depression symptoms [22,25]. Three RCTs reported data on quality of life $(n=826)[22,23,25]$. Participants receiving duloxetine reported statistically significant benefits on quality of life outcomes versus the placebo groups (SMD, o.40; 95\% CI, 0.26 to 0.53 ), with minimal heterogeneity observed between studies.

Discontinuations due to adverse events All five included RCTs reported discontinuation due to adverse event (DAE, $\mathrm{n}=1,772$ ). Individuals receiving duloxetine were twice as likely to discontinue during the 


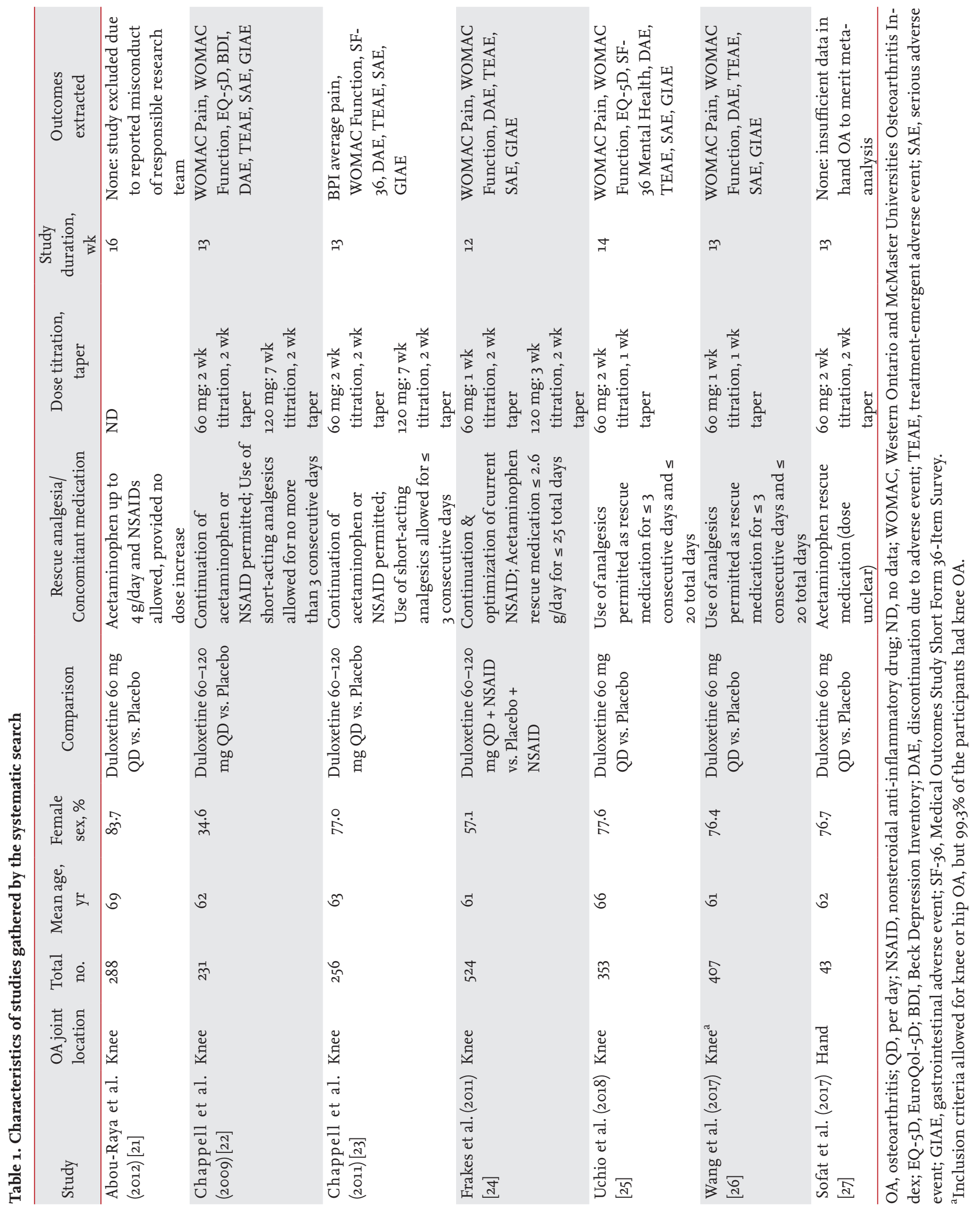


study period due to an adverse event (RR, 2.17; 95\% CI, 1.57 to 3.01). Heterogeneity between the study estimates was assessed to be negligible.

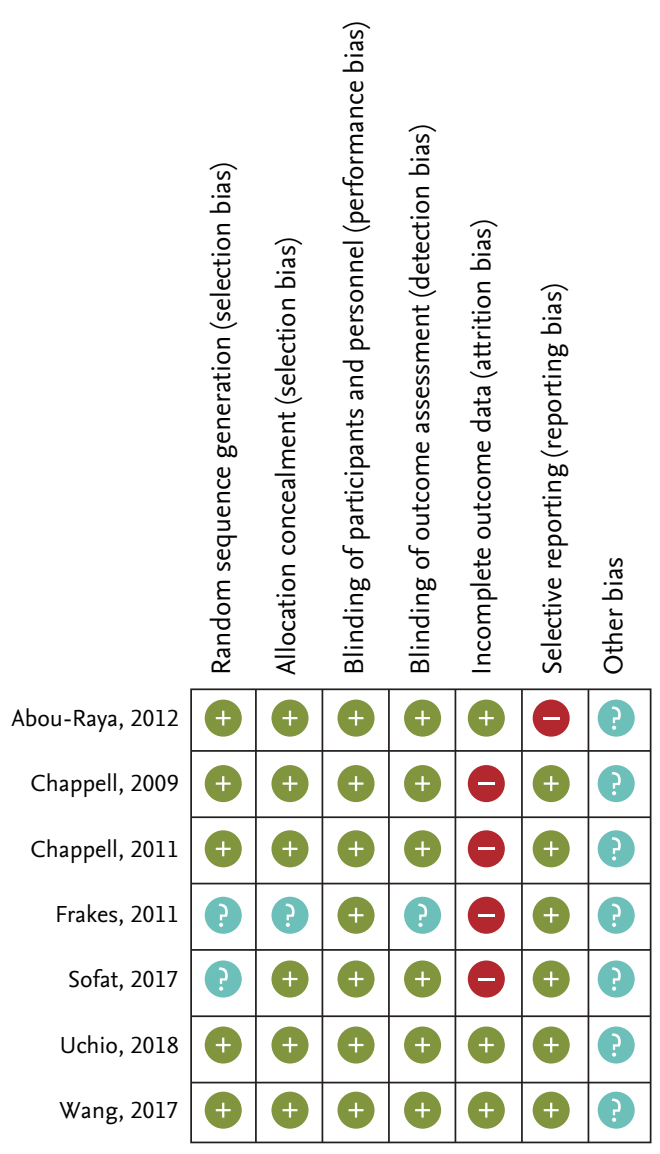

Figure 2. Risk of bias summary.

Random sequence generation (selection bias)

Allocation concealment (selection bias)

Blinding of participants and personnel (performance bias)

Blinding of outcome assessment (detection bias)

Incomplete outcome data (attrition bias)

Low risk of bias

Unclear risk of bias

High risk of bias

Figure 3. Risk of bias distribution.

\section{Selective reporting (reporting bias)}

Other bias

\section{TEAEs and SAEs}

Five studies reported TEAEs and SAEs $(n=1,762)$. TEAEs were about $50 \%$ more likely to occur in participants receiving duloxetine ( $\mathrm{RR}, 1.53 ; 95 \% \mathrm{CI}, 1.21$ to 1.92). The rates of SAEs were equal between the two groups, with no statistically significant differences observed.

\section{Gastrointestinal adverse events}

Among the five RCTs $(n=1,762)$ that reported on gastrointestinal adverse events (GIAEs), participants receiving duloxetine were nearly 4.5 times more likely to experience an event (RR, 4.43; 95\% CI, 3.45 to 5.69). Heterogeneity between the studies was negligible. The most commonly reported GIAE were nausea, constipation, and dry mouth.

\section{Efficacy of duloxetine in hand $O A$}

The RCT ( $n=43$ ) assessing the efficacy of duloxetine in hand OA patients reported no statistically significant differences between the duloxetine and placebo groups with regard to pain, function, or depression outcomes [27]. The proportion of participants experiencing GIAE was over three times higher in the duloxetine group than in the placebo group in this study. Given the small sample size, no definitive conclusions can be drawn for all hand OA patients from these results.

\section{DISCUSSION}

The results of our study indicate that duloxetine has

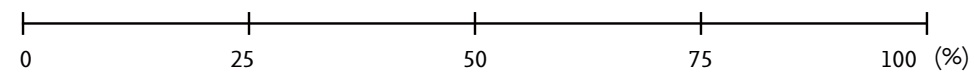


Table 2. Meta-analysis results

\begin{tabular}{|c|c|c|c|}
\hline Outcome & No. of RCTs & No. of patients & Effect estimate (95\% CI) \\
\hline Pain [22-26] & 5 & 1,713 & $\mathrm{SMD}-0.38(-0.48$ to -0.28$) ; I^{2}=5 \%, T^{2}=0$ \\
\hline Function $[22-26]$ & 5 & 1,695 & $\mathrm{SMD}-0.35(-0.46$ to -0.24$) ; I^{2}=23 \%, T^{2}=0$ \\
\hline Depression $[22,25]$ & 2 & 584 & SMD $-0.09(-0.26$ to 0.07$) ; I^{2}=0 \%, T^{2}=0$ \\
\hline Quality of life $[22,23,25]$ & 3 & 826 & $\operatorname{SMD} 0.40(0.26$ to 0.53$) ; I^{2}=0 \%, T^{2}=0$ \\
\hline Discontinuation due to adverse event(s), \% [22-26] & 5 & 1,772 & $\begin{array}{l}\text { RR } 2.17 \text { (1.57 to 3.01); } I^{2}=0 \%, T^{2}=0 \\
\text { (duloxetine: } 12.4 \% \text {; placebo: } 5.5 \% \text { ) }\end{array}$ \\
\hline Treatment-emergent adverse event(s), \% [22-26] & 5 & 1,762 & $\begin{array}{l}\text { RR } 1.53(1.21 \text { to } 1.92) ; I^{2}=77 \%, T^{2}=0.05 \\
\text { (duloxetine: } 55.1 \% \text {; placebo: } 37.4 \% \text { ) }\end{array}$ \\
\hline Serious adverse event(s), \% [22-26] & 5 & 1,762 & $\begin{array}{l}\text { RR } 1.03(0.42 \text { to } 2.54) ; I^{2}=0 \% T^{2}=0 \\
\text { (duloxetine: } 1.1 \% ; \text { placebo: } 1.2 \% \text { ) }\end{array}$ \\
\hline Gastrointestinal adverse event(s), \% [22-26] & 5 & 1,762 & $\begin{array}{l}\text { RR } 4.43(3.45 \text { to } 5.69) ; I^{2}=4 \%, T^{2}=0 \\
\text { (duloxetine: } 35.5 \% ; \text { placebo: } 7.7 \% \text { ) }\end{array}$ \\
\hline
\end{tabular}

statistically significant, moderate benefits on pain and function in knee OA patients for up to 3 months. The effect sizes observed for pain and functional improvement were comparable to those reported for prescription doses of NSAIDs for knee OA [29]. Despite the potential benefits of duloxetine, reported incidences of GIAE were three to four times higher in participants with both knee and hand OA who received the intervention versus placebo.

Our results are consistent with previous meta-analyses of duloxetine in knee OA, despite the addition of two new RCTs [30,31]. Previous meta-analyses have reported statistically significant benefits of the drug on pain and function, with statistically significantly higher incidences of TEAEs and DAEs.

Interestingly, the results of our study on depression are also consistent with the most recent meta-analyses that compared duloxetine with other antidepressants $[32,33]$. The authors of a 2012 Cochrane review concluded that duloxetine was not an appropriate first line treatment for major depressive disorder due to its unfavorable adverse event profile and high cost. Duloxetine was not highly ranked with regard to efficacy in mitigating depressive symptoms in either review, and both reviews highlighted its high potential for adverse GI side effects. Our study showed that while duloxetine demonstrated moderate and significant benefits on knee OA symptoms over the course of 13 weeks, the observed effect sizes were not superior to those that are observed in meta-analyses of conventional treatments such as NSAIDs. Additionally, the adverse event profile was similar to that of NSAIDs, particularly with regard to disproportionately more DAE in the intervention group and the incidence of GIAE. It is important to note; however, that the most commonly reported GIAEs among duloxetine users were minor and transient and included nausea, constipation, and decreased appetite. Longer term safety data are required to determine whether duloxetine could be a viable alternative to NSAIDs with regard to the reduction of risk for serious GIAEs such as intestinal bleeding and ulceration [34].

Duloxetine demonstrated significant beneficial effects on quality of life in knee OA patients. A recent cost-utility analysis highlighted the specific benefits of duloxetine on quality of life and as such, ranked it more favorably than NSAIDs and opioid medications; these results were particularly applicable to patients who are more susceptible to NSAID-specific adverse events [35]. Despite the specific intention of duloxetine to act on depressive symptoms, use of the drug did not result in statistically significant improvement of symptoms of depression in any of the included RCTs in OA patients. The reason for this may be that in most of the included RCTs, individuals with psychiatric disorders or major depressive disorders were excluded.

Quality assessment and the results of our meta-analysis of DAE suggest a potential for bias due to differential rates of DAE. A methodological analysis of duloxetine trials in chronic pain populations highlighted differential DAE rates in participants receiving the drug 
and concluded that imputation methods using the last observation carried forward (LOCF) method could inflate the observed treatment effect of duloxetine in clinical trials [20]. We acknowledge that such bias may have impacted the results of our study, with the overall effect sizes for pain and function being potentially biased in favor of duloxetine.

\section{CONCLUSIONS}

The results of our study indicate that duloxetine may be an effective treatment option for individuals with OA, but that use of the drug is associated with a significantly higher risk of adverse events. Patient preferences, cost considerations, and clinicians' judgment must be taken into account before the initiation of a duloxetine regimen. Future RCTs should be conducted in patients who have concomitant OA and depression to assess the specific benefits of duloxetine in these populations, and to address the real-world scenario in which duloxetine may be a more favorable option. Studies focused on the safety of long-term use of the drug should also be conducted, to assess its eligibility as an alternative to conventional treatments that are associated with a risk of SAEs with long-term use.

\section{Conflict of interest}

No potential conflict of interest relevant to this article was reported.

\section{REFERENCES}

1. Johannes CB, Le TK, Zhou X, Johnston JA, Dworkin RH. The prevalence of chronic pain in United States adults: results of an Internet-based survey. J Pain 2010;11:12301239.

2. Rustoen T, Wahl AK, Hanestad BR, Lerdal A, Paul S, Miaskowski C. Age and the experience of chronic pain: differences in health and quality of life among younger, middle-aged, and older adults. Clin J Pain 2005;21:513-523.

3. Fransen M, Bridgett L, March L, Hoy D, Penserga E, Brooks P. The epidemiology of osteoarthritis in Asia. Int J Rheum Dis 2011;14:113-121.

4. Kim I, Kim HA, Seo YI, et al. Tibiofemoral osteoarthritis affects quality of life and function in elderly Koreans, with women more adversely affected than men. BMC Musculoskelet Disord 2010;11:129.

5. Arendt-Nielsen L, Nie H, Laursen MB, et al. Sensitization in patients with painful knee osteoarthritis. Pain 2010;149:573-581.

6. Bajaj P, Bajaj P, Graven-Nielsen T, Arendt-Nielsen L. Osteoarthritis and its association with muscle hyperalgesia: an experimental controlled study. Pain 2001;93:107-114.

7. Kidd BL. Osteoarthritis and joint pain. Pain 2006;123:6-9.

8. Havelin J, Imbert I, Cormier J, Allen J, Porreca F, King T. Central sensitization and neuropathic features of ongoing pain in a rat model of advanced osteoarthritis. J Pain 2016;17:374-382.

9. Agarwal P, Sambamoorthi U. Healthcare expenditures associated with depression among individuals with osteoarthritis: post-regression linear decomposition approach. J Gen Intern Med 2015;30:1803-1811.

10. Sale JE, Gignac M, Hawker G. The relationship between disease symptoms, life events, coping and treatment, and depression among older adults wi th osteoarthritis. J Rheumatol 2008;35:335-342.9.

11. Moher D, Liberati A, Tetzlaff J, Altman DG; PRISMA Group. Preferred reporting items for systematic reviews and meta-analyses: the PRISMA statement. PLoS Med 2009;6:e1000097.

12. Review Manager (RevMan) (Computer program) version 5.3 [Internet]. Copenhagen (DK): The Nordic Cochrane Centre, The Cochrane Collaboration, 2014 [cited 2019 Jan 30]. Available from: https://community.cochrane.org/help/ tools-and-software/revman-5.

13. The Cochrane Risk of Bias Tool. Copenhagen (DK): The Nordic Cochrane Centre, The Cochrane Collaboration, 2014 [cited 2019 Jan 30]. Available from: https://sites.google.com/site/riskofbiastool.

14. Khawam EA, Laurencic G, Malone DA Jr. Side effects of antidepressants: an overview. Cleve Clin J Med 2006;73:351353.

15. U.S. Food and Drug Administration. CYMBALTA (duloxetine hydrochloride) delayed-release Capsules [Internet]. Silver Spring (MD): FDA, 2007 [cited 2019 Jan 30]. Available from: https://www.accessdata.fda.gov/drugsatfda_ docs/label/2007/021427soo9so11so13lbl.pdf.

16. DerSimonian R, Laird N. Meta-analysis in clinical trials. Control Clin Trials 1986;7:177-188.

17. Mantel N, Haenszel W. Statistical aspects of the analysis 
of data from retrospective studies of disease. J Natl Cancer Inst 1959;22:719-748.

18. Rucker G, Schwarzer G, Carpenter JR, Schumacher M. Undue reliance on $\mathrm{I}(2)$ in assessing heterogeneity may mislead. BMC Med Res Methodol 2008;8:79.

19. Higgins JP, Thompson SG. Quantifying heterogeneity in a meta-analysis. Stat Med 2002;21:1539-1558.

20. Moore RA, Cai N, Skljarevski V, Tolle TR. Duloxetine use in chronic painful conditions: individual patient data responder analysis. Eur J Pain 2014;18:67-75.

21. Abou-Raya S, Abou-Raya A, Helmii M. Duloxetine for the management of pain in older adults with knee osteoarthritis: randomised placebo-controlled trial. Age Ageing 2012;41:646-652.

22. Chappell AS, Ossanna MJ, Liu-Seifert H, et al. Duloxetine, a centrally acting analgesic, in the treatment of patients with osteoarthritis knee pain: a 13-week, randomized, placebo-controlled trial. Pain 2009;146:253-260.

23. Chappell AS, Desaiah D, Liu-Seifert H, et al. A double-blind, randomized, placebo-controlled study of the efficacy and safety of duloxetine for the treatment of chronic pain due to osteoarthritis of the knee. Pain Pract 2011;11:33-41.

24. Frakes EP, Risser RC, Ball TD, Hochberg MC, Wohlreich MM. Duloxetine added to oral nonsteroidal anti-inflammatory drugs for treatment of knee pain due to osteoarthritis: results of a randomized, double-blind, placebo-controlled trial. Curr Med Res Opin 2011;27:2361-2372.

25. Uchio Y, Enomoto H, Alev L, et al. A randomized, double-blind, placebo-controlled Phase III trial of duloxetine in Japanese patients with knee pain due to osteoarthritis. J Pain Res 2018;11:809-821.

26. Wang G, Bi L, Li X, et al. Efficacy and safety of duloxetine in Chinese patients with chronic pain due to osteoar- thritis: a randomized, double-blind, placebo-controlled study. Osteoarthritis Cartilage 2017;25:832-838.

27. Sofat N, Harrison A, Russell MD, et al. The effect of pregabalin or duloxetine on arthritis pain: a clinical and mechanistic study in people with hand osteoarthritis. J Pain Res 2017;10:2437-2449.

28. Abou-Raya A, Abou-Raya S, Khadrawe T. Retracted: methotrexate in the treatment of symptomatic knee osteoarthritis: randomised placebo-controlled trial. Ann Rheum Dis 2018;77:e46.

29. da Costa BR, Reichenbach S, Keller N, et al. Effectiveness of non-steroidal anti-inflammatory drugs for the treatment of pain in knee and hip osteoarthritis: a network meta-analysis. Lancet 2017;390:e21-e33.

30. Wang ZY, Shi SY, Li SJ, et al. Efficacy and safety of duloxetine on osteoarthritis knee pain: a meta-analysis of randomized controlled trials. Pain Med 2015;16:1373-1385.

31. Ananias J, Irarrazaval S. Is duloxetine an alternative in the treatment of osteoarthritis? Medwave 2017;17:e7063.

32. Cipriani A, Koesters M, Furukawa TA, et al. Duloxetine versus other anti-depressive agents for depression. Cochrane Database Syst Rev 2012;10:CDoo6533.

33. Cipriani A, Furukawa TA, Salanti G, et al. Comparative efficacy and acceptability of 21 antidepressant drugs for the acute treatment of adults with major depressive disorder: a systematic review and network meta-analysis. Lancet 2018;391:1357-1366.

34. Sostres C, Gargallo CJ, Lanas A. Nonsteroidal anti-inflammatory drugs and upper and lower gastrointestinal mucosal damage. Arthritis Res Ther 2013;15 Suppl 3:S3.

35. Wielage RC, Patel AJ, Bansal M, Lee S, Klein RW, Happich M. Cost effectiveness of duloxetine for osteoarthritis: a Quebec societal perspective. Arthritis Care Res (Hoboken) 2014;66:702-708. 


\section{Appendix 1. MEDLINE search strategy}

1. duloxetine.mp. [mp=title, abstract, original title, name of substance word, subject heading word, floating sub-heading word, keyword heading word, protocol supplementary concept word, rare disease supplementary concept word, unique identifier, synonyms]

2. exp osteoarthritis/ or osteoarthriti\$.ti,ab. or osteoarthro\$.ti,ab. or gonarthriti\$.ti,ab. or gonarthro\$.ti,ab. or coxarthriti\$.ti,ab. or coxarthro\$.ti,ab. or arthros\$.ti,ab. or arthrot\$.ti,ab.

3. exp randomized controlled trials/

4. exp random allocation/

5. exp double-blind method/

6. exp single blind method/

7. exp cross-over studies/

8. exp clinical trial/

9. randomized controlled trial.pt.

10. controlled clinical trial.pt.

11. (random $\$$ or rct).tw.

12. (clin\$ adj25 trial\$).tw.

13. ((singl\$ or doubl\$ or trebl\$ or tripl\$) adj (blind $\$$ or mask\$)).tw.

14. 3 or 4 or 5 or 6 or 7 or 8 or 9 or 10 or 11 or 12 or 13

15. 1 and 2 and 14

16. limit 15 to humans

17. remove duplicates from 16 
Supplementary Table 1. PRISMA 2009 checklist

\begin{tabular}{|c|c|c|c|}
\hline Section/topic & \# & Checklist item & $\begin{array}{l}\text { Reported on } \\
\text { page \# }\end{array}$ \\
\hline \multicolumn{4}{|l|}{ Title } \\
\hline Title & 1 & Identify the report as a systematic review, meta-analysis, or both. & 1 \\
\hline \multicolumn{4}{|l|}{ Abstract } \\
\hline Structured summary & 2 & $\begin{array}{l}\text { Provide a structured summary including, as applicable: } \\
\text { background; objectives; data sources; study eligibility criteria, } \\
\text { participants, and interventions; study appraisal and synthesis } \\
\text { methods; results; limitations; conclusions and implications of } \\
\text { key findings; systematic review registration number. }\end{array}$ & 2 \\
\hline \multicolumn{4}{|l|}{ Introduction } \\
\hline Rationale & 3 & $\begin{array}{l}\text { Describe the rationale for the review in the context of what is } \\
\text { already known. }\end{array}$ & 3 \\
\hline Objectives & 4 & $\begin{array}{l}\text { Provide an explicit statement of questions being addressed with } \\
\text { reference to participants, interventions, comparisons, outcomes, } \\
\text { and study design (PICOS). }\end{array}$ & 3 \\
\hline \multicolumn{4}{|l|}{ Methods } \\
\hline Protocol and registration & 5 & $\begin{array}{l}\text { Indicate if a review protocol exists, if and where it can be accessed } \\
\text { (e.g., Web address), and, if available, provide registration } \\
\text { information including registration number. }\end{array}$ & No \\
\hline Eligibility criteria & 6 & $\begin{array}{l}\text { Specify study characteristics (e.g., PICOS, length of follow-up) } \\
\text { and report characteristics (e.g., years considered, language, } \\
\text { publication status) used as criteria for eligibility, giving rationale. }\end{array}$ & $3-4$ \\
\hline Information sources & 7 & $\begin{array}{l}\text { Describe all information sources (e.g., databases with dates } \\
\text { of coverage, contact with study authors to identify additional } \\
\text { studies) in the search and date last searched. }\end{array}$ & 3 \\
\hline Search & 8 & $\begin{array}{l}\text { Present full electronic search strategy for at least one database, } \\
\text { including any limits used, such that it could be repeated. }\end{array}$ & $\begin{array}{l}\text { Supplemental } \\
\text { Table } 1\end{array}$ \\
\hline Study selection & 9 & $\begin{array}{l}\text { State the process for selecting studies (i.e., screening, eligibility, } \\
\text { included in systematic review, and, if applicable, included in the } \\
\text { meta-analysis). }\end{array}$ & 4 \\
\hline Data collection process & 10 & $\begin{array}{l}\text { Describe method of data extraction from reports (e.g., piloted } \\
\text { forms, independently, in duplicate) and any processes for } \\
\text { obtaining and confirming data from investigators. }\end{array}$ & 4 \\
\hline Data items & 11 & $\begin{array}{l}\text { List and define all variables for which data were sought } \\
\text { (e.g., PICOS, funding sources) and any assumptions and } \\
\text { simplifications made. }\end{array}$ & $4-5$ \\
\hline $\begin{array}{l}\text { Risk of bias in individual } \\
\text { studies }\end{array}$ & 12 & $\begin{array}{l}\text { Describe methods used for assessing risk of bias of individual } \\
\text { studies (including specification of whether this was done at the } \\
\text { study or outcome level), and how this information is to be used } \\
\text { in any data synthesis. }\end{array}$ & 4 \\
\hline Summary measures & 13 & $\begin{array}{l}\text { State the principal summary measures (e.g., risk ratio, difference } \\
\text { in means). }\end{array}$ & 5 \\
\hline Synthesis of results & 14 & $\begin{array}{l}\text { Describe the methods of handling data and combining results of } \\
\text { studies, if done, including measures of consistency }\left(\text { e.g., } I^{2}\right) \text { for } \\
\text { each meta-analysis. }\end{array}$ & 5 \\
\hline Risk of bias across studies & 15 & $\begin{array}{l}\text { Specify any assessment of risk of bias that may affect the } \\
\text { cumulative evidence (e.g., publication bias, selective reporting } \\
\text { within studies). }\end{array}$ & 5 \\
\hline
\end{tabular}




\section{KJIM}

\section{Supplementary Table 1. Continued}

\begin{tabular}{|c|c|c|c|}
\hline Section/topic & $\#$ & Checklist item & $\begin{array}{l}\text { Reported on } \\
\text { page \# }\end{array}$ \\
\hline Additional analyses & 16 & $\begin{array}{l}\text { Describe methods of additional analyses (e.g., sensitivity or } \\
\text { subgroup analyses, meta-regression), if done, indicating which } \\
\text { were pre-specified. }\end{array}$ & 5 \\
\hline \multicolumn{4}{|l|}{ Results } \\
\hline Study selection & 17 & $\begin{array}{l}\text { Give numbers of studies screened, assessed for eligibility, and } \\
\text { included in the review, with reasons for exclusions at each stage, } \\
\text { ideally with a flow diagram. }\end{array}$ & 5-6, Figure 1 \\
\hline Study characteristics & 18 & $\begin{array}{l}\text { For each study, present characteristics for which data were } \\
\text { extracted (e.g., study size, PICOS, follow-up period) and provide } \\
\text { the citations. }\end{array}$ & 5-6, Table 1 \\
\hline Risk of bias within studies & 19 & $\begin{array}{l}\text { Present data on risk of bias of each study and, if available, any } \\
\text { outcome level assessment (see item 12). }\end{array}$ & Table 2 \\
\hline Results of individual studies & 20 & $\begin{array}{l}\text { For all outcomes considered (benefits or harms), present, for each } \\
\text { study: (a) simple summary data for each intervention group (b) } \\
\text { effect estimates and confidence intervals, ideally with a forest } \\
\text { plot. }\end{array}$ & 6-8, Table 3 \\
\hline Synthesis of results & 21 & $\begin{array}{l}\text { Present results of each meta-analysis done, including confidence } \\
\text { intervals and measures of consistency. }\end{array}$ & 6-8, Table 3 \\
\hline Risk of bias across studies & 22 & $\begin{array}{l}\text { Present results of any assessment of risk of bias across studies (see } \\
\text { Item 15). }\end{array}$ & 6, Figure 2 \\
\hline Additional analysis & 23 & $\begin{array}{l}\text { Give results of additional analyses, if done (e.g., sensitivity or } \\
\text { subgroup analyses, meta-regression [see Item 16]). }\end{array}$ & $6-7$ \\
\hline \multicolumn{4}{|l|}{ Discussion } \\
\hline Summary of evidence & 24 & $\begin{array}{l}\text { Summarize the main findings including the strength of evidence } \\
\text { for each main outcome; consider their relevance to key groups } \\
\text { (e.g., healthcare providers, users, and policy makers). }\end{array}$ & $8-9$ \\
\hline Limitations & 25 & $\begin{array}{l}\text { Discuss limitations at study and outcome level (e.g., risk of } \\
\text { bias), and at review-level (e.g., incomplete retrieval of identified } \\
\text { research, reporting bias). }\end{array}$ & $9-10$ \\
\hline Conclusions & 26 & $\begin{array}{l}\text { Provide a general interpretation of the results in the context of } \\
\text { other evidence, and implications for future research. }\end{array}$ & 10 \\
\hline \multicolumn{4}{|l|}{ Funding } \\
\hline Funding & 27 & $\begin{array}{l}\text { Describe sources of funding for the systematic review and other } \\
\text { support (e.g., supply of data); role of funders for the systematic } \\
\text { review. }\end{array}$ & 11 \\
\hline
\end{tabular}

Adapted from Moher et al. [11]. For more information, visit: www.prisma-statement.org.

PRISMA, Preferred Reporting Items for Systematic Reviews and Meta-Analyses. 